\title{
Recent results from the KASCADE-Grande data analysis
}

D. Kang ${ }^{1 *}$, W.D. Apel ${ }^{1}, J . C$. Arteaga-Velázquez ${ }^{2}, K$. Bekk $^{1}, M$. Bertaina $^{3}, J$. Blümer $^{1,4 * *}, H$. Bozdog $^{1}, I . M$. Brancus $^{5 * * *}, E$. Cantoni $^{3,6}, A$. Chiavassa $^{3}, F$. Cossavella ${ }^{4}, K$. Daumiller ${ }^{1}, V$. de Souza ${ }^{7}, F$. Di Pierro ${ }^{3}, P$. Doll ${ }^{1}, R$. Engel ${ }^{1}, D$. Fuhrmann $^{8}, A$. Gherghel-Lascu $^{5}$, H.J. Gils ${ }^{1}, R$. Glasstetter ${ }^{8}, C$. Grupen ${ }^{9}, A$. Haungs $^{1}$, D. Heck ${ }^{1}, J . R$. Hörandel ${ }^{10}, D$. Huber $^{4}, T$. Huege ${ }^{1}$,

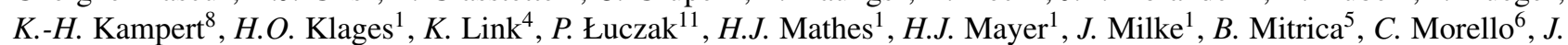
Oehlschläger ${ }^{1}, S$. Ostapchenko ${ }^{12}, N$. Palmieri ${ }^{4}, T$. Pierog ${ }^{1}, H$. Rebel ${ }^{1}, M$. Roth ${ }^{1}, H$. Schieler ${ }^{1}, S$. Schoo $^{1}, F . G$. Schröder $^{1}$,

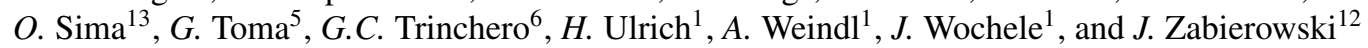

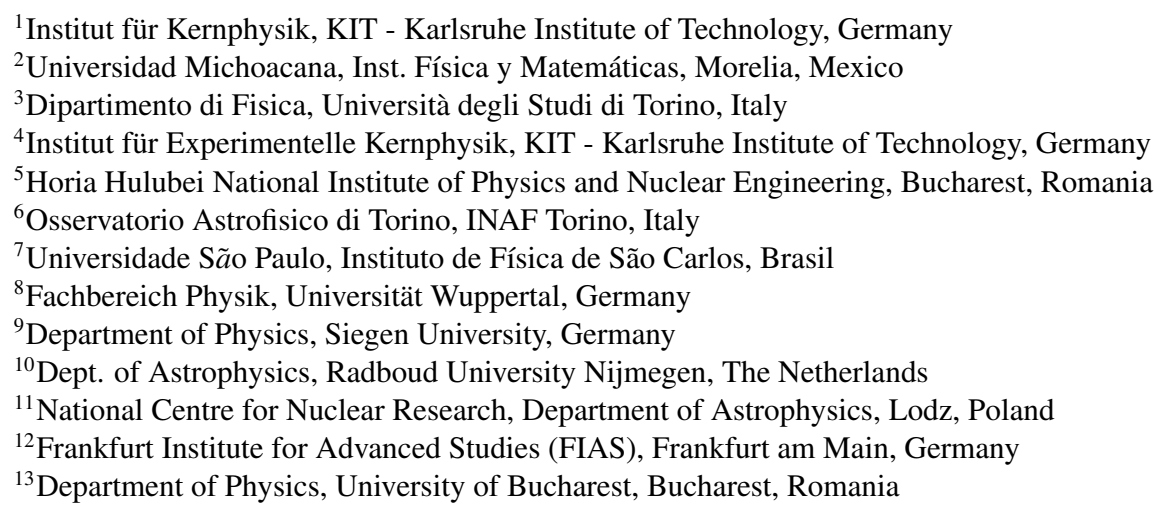

\begin{abstract}
KASCADE, together with its extension KASCADE-Grande measured individual air showers of cosmic rays in the primary energy range of $100 \mathrm{TeV}$ to $1 \mathrm{EeV}$. The data collection was fully completed at the end of 2013 and the experiment was dismantled. However, the data analysis is still in progress. Recently, we published a new result on upper limits to the flux of ultra-high energy gamma rays, which set constraints on some fundamental astrophysical models. We also use the data to investigate the validity of the new hadronic interactions models like SIBYLL version 2.3c or EPOS-LHC. In addition, we updated and improved the webbased platform of the KASCADE Cosmic Ray Data Centre (KCDC), where now the data from KASCADE and KASCADE-Grande of more than 20 years measurements is available, including corresponding Monte-Carlo simulated events based on three different hadronic interaction models. In this contribution, recent results from KASCADE-Grande and the update of KCDC is briefly discussed.
\end{abstract}

\section{Introduction}

Investigations of high-energy cosmic rays are devoted to understand the mass composition, the energy spectrum, and the arrival direction of cosmic rays. These studies are important to grasp the origin of cosmic rays, as well as their propagation and acceleration. In particular, measurements in the energy range of $\mathrm{PeV}$ to EeV covered by KASCADE and KASCADE-Grande give a clue to identify the transition region of galactic and extragalactic cosmic rays.

Extensive air shower arrays of KASCADE [1] and its extension KASCADE-Grande [2] were located at the Karlsruhe Institute of Technology, Karlsruhe, Germany (49.1 $1^{\circ}$ north, $8.4^{\circ}$ east, $110 \mathrm{~m}$ above sea level). The data accumulation was completed at the end of 2013 and all detector components are fully dismantled. Detailed anal-

\footnotetext{
*e-mail: donghwa.kang@kit.edu

**Now: Head of KIT Division V - Physics and Mathematics

$* * *$ deceased
}

ysis of more than 20 years measured data presents fruitful results: The all-particle energy spectrum reconstructed using the KASCADE data shows a knee-like structure due to a steepening of spectra of light elements [3]. The allparticle energy spectrum measured by KASCADE-Grande [4] shows some structures, which cannot described by a single power law: a concave behavior just above $10^{16} \mathrm{eV}$ and a small break at around $10^{17} \mathrm{eV}$, where a knee-like feature would be expected as the knee of the heavy primaries, mainly iron. In the reconstructed energy spectra for individual mass groups, the knee-like feature in the heavy primary spectrum is observed much more significantly at around $80 \mathrm{PeV}$ [5]. Further, an ankle-like structure is observed at an energy of $100 \mathrm{PeV}$ in the energy spectrum of light primary cosmic rays [6].

The analysis of the measured data in more than 20 years is still in progress. In this paper, we will discuss the recently ongoing studies: the combined analysis, testing of the new interaction model of SIBYLL 2.3c, and the 
limit on the diffuse gamma-ray flux. Finally, the KASCADE Cosmic ray Data Center (KCDC) will be discussed as well.

\section{Combined analysis}

Historically, the analysis of the measured KASCADE and KASCADE-Grande data was performed independently. Thus the main goal for a combined analysis is to get the coherent energy spectrum by one consistent reconstruction procedure, and also its mass composition from $10^{14}$ up to $10^{18} \mathrm{eV}$. This combined analysis was done by means of extending the fiducial area and a higher accuracy of the shower reconstruction, in order to scrutinize earlier observations of spectral features. For the determination of primary energy, we used the $k$ parameter method, which is the normalized shower size ratio [4]. In addition, using this $k$ parameter, we separated the data sets in light and heavy components, since the $k$ value close to zero is valid for the proton primary and close to one for iron primary. It has to be noted that the electron number is used in the combined analysis instead of the number of charged particles. The accuracy should be comparable anyway, since the electromagnetic component is dominant in the relevant energy range.

The resulting energy spectra based on post-LHC models (QGSJetII-04, EPOS-LHC, SIBYLL2.3) can be found in Ref. [7]. All features observed by the separate analysis are well confirmed. In the comparison of the energy spectra based on the QGSJetII-04 and EPOS-LHC models, light primary interactions agree well each other, but heavy primary interactions show some differences with increasing energies. The reason might be originally in the muon components [8]. Studies on the muon number is under investigation.

\section{Test of hadronic interaction model}

One of the important analysis after completeness is the test of hadronic interaction models with KASCADE and KASCADE-Grande data. Recently, a new version of SIBYLL 2.3c is developed [9]. By means of the shower size measured by KASCADE-Grande data, initial tests of SIBYLL 2.3c were performed. Figure 1 (top) shows the shower size measured by KASCADE-Grande, including the full detector response by simulation, along with proton and iron induced showers for the QGSJetII-04, EPOSLHC, SIBYLL 2.3 and SIBYLL 2.3c simulations. The solid symbols are for proton induced showers and open ones are for iron, predicted by different interaction models. SIBYLL 2.3c has a similar tendency to the SIBYLL 2.3 model, but it has less muons compared to EPOS-LHC. A ratio of the total number of charged particles $\left(N_{c h}\right)$ to the total muon numbers $\left(N_{\mu}\right)$ for different interaction models in the bottom plot in Fig. 2. Both QGSJetII-04 and SIBYLL 2.3 models have a similar abundance ratio of $N_{c h}$ to $N_{\mu}$, but EPOS-LHC has approximately $10 \%$ more muons, and SIBYLL 2.3c has about 10\% less muons, comparing to QGSJetII-04. It implies that a less dominant light
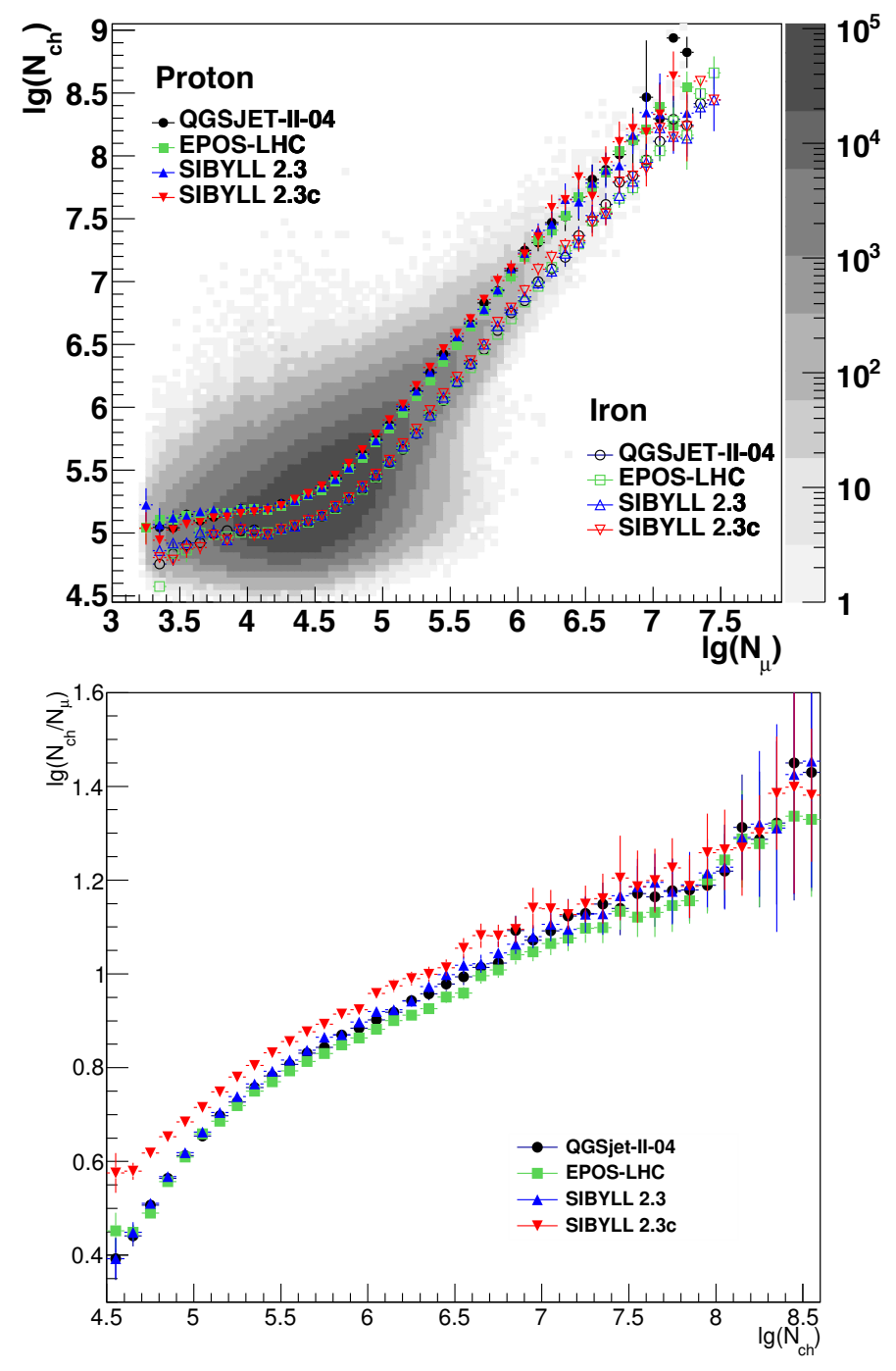

Figure 1. The 2-dim. shower size distribution measured by KASCADE-Grande, along with proton and iron induced showers for different simulations (top). The ratio of the total number of charged particles to the total muon numbers (bottom).

mass composition is predicted if SIBYLL 2.3c is used to reconstruct the primary mass.

Based on the shower size measured only by KASCADE-Grande, we reconstructed the primary energy spectrum by the energy calibration with the new SIBYLL $2.3 \mathrm{c}$ model. To reconstruct spectra for individual mass groups, we divided two subsets of data for heavy and light groups, based on the $y$ cut method [10]. The energy calibration function for light and heavy induced showers is shown in Fig. 2 (top). The slope of SIBYLL 2.3c is quite different from the other two models, and interestingly two lines meet at energies about $10^{18} \mathrm{eV}$. Using this fit function, we converted the attenuation corrected shower size into the reconstructed energy. Figure 2 (bottom) presents the reconstructed energy spectra for light and heavy induced showers. Interestingly, the spectrum for light primaries is very close to the one for heavy primary at energies around $1 \mathrm{EeV}$. Compared to other previous SIBYLL models, we 

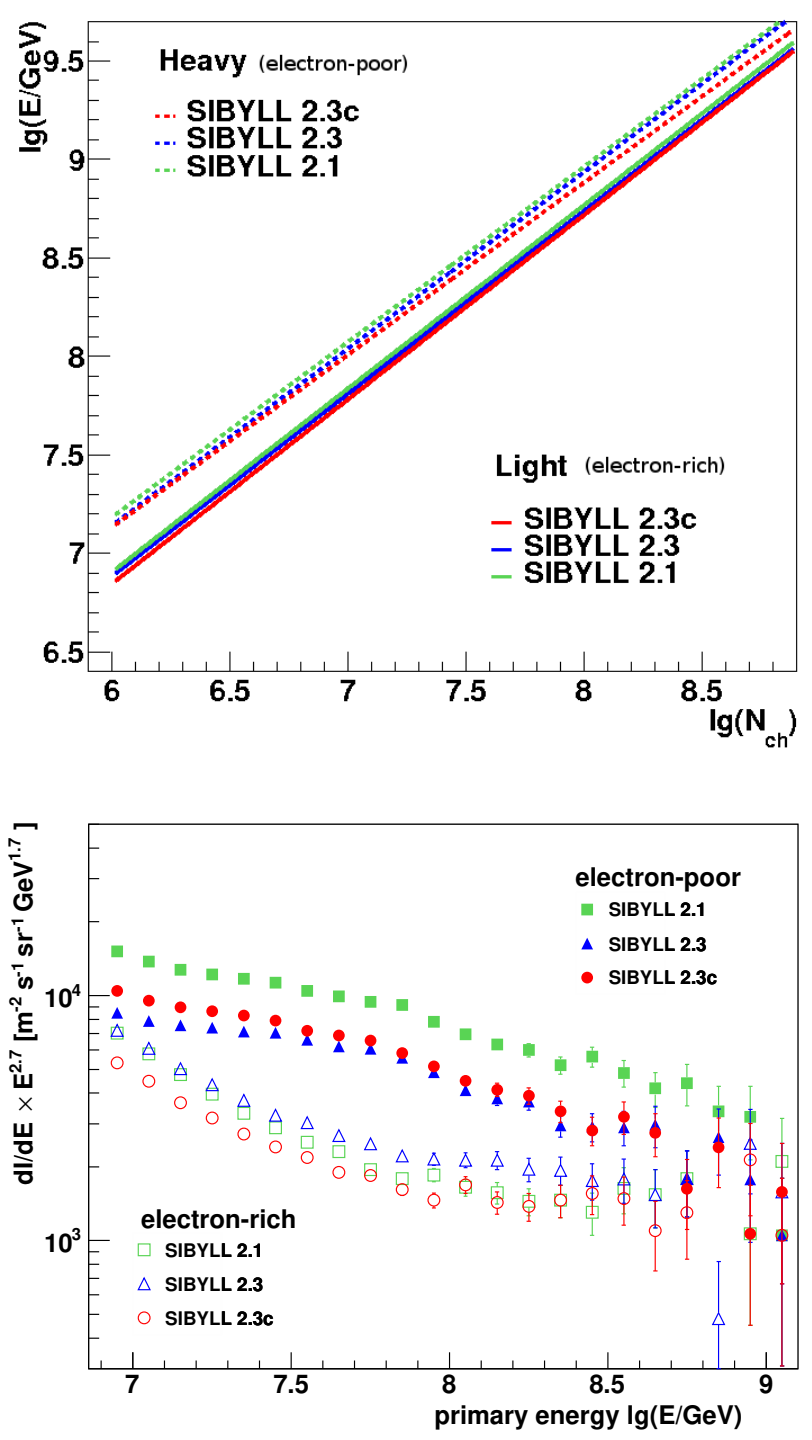

Figure 2. The energy calibration function of light and heavy primaries for SIBYLL 2.3c, SIBYLL 2.3, and SIBYLL (top) and the resulting energy spectra based on the three different models (bottom).

see a slight discrepancy of the spectral slopes, due to the different ratio of $N_{c h} / N_{\mu}$, but all the spectra show a similar feature. The total flux is shifted about $10-20 \%$, but the general structure are similar.

\section{Limits on diffuse gamma-ray flux}

The flux of the diffuse gamma rays, its spectrum and its evolution are very interesting topics in astrophysics. This study gives information about processes and developments at large distance from our Galaxy. We can understand the origin and the propagation of galactic cosmic rays from investigations of the galactic diffuse emission. Gamma-ray induced air showers are notable for their lack of muons, compared to hadronic showers. Hence, we select a sample greatly enriched in photon showers by rejecting showers containing muons.

The selection of muon-poor showers was found and optimized by the Monte-Carlo simulations in order to maximize the purity/efficiency ratio of gamma-ray induced events. The events after the selection are expected to be mainly due to primary photons because air showers induced by heavy nuclei show a larger muon to electron ratio. We assumed thus that all these events are primary gamma-rays and set upper limits on the gamma-ray fraction of the cosmic rays. We estimated the $90 \%$ C.L. upper limit on the number of detected events, using standard statistical methods [11] and use the simulation data to evaluate the efficiency for gamma-ray detection, when the muon cut is applied.

Figure 3 (top) displays the measurements on the gamma-ray fraction as a function of the energy, including this work, for the energy range of $10^{14} \mathrm{eV}$ up to $10^{18} \mathrm{eV}$. The upper limit of the fraction of gamma-rays at $1.5 \cdot 10^{15}$ $\mathrm{eV}$ and $3.7 \cdot 10^{15} \mathrm{eV}$ are obtained to be $1.7 \cdot 10^{5}$ and $1.1 \cdot 10^{5}$, respectively. These are the lowest upper limits up to now. In addition, as around $10^{17} \mathrm{eV}$ not many experiments have reported results, the limits obtained by KASCADE-Grande are of prominent interest. It should be noted that all values in Fig. 3 are upper limits, except the one from MSU.

Figure 3 (bottom) shows the comparison of integral flux of gamma rays with other previous experiments. Compared with the earlier published limits by KASCADE in 2003 [12] there are only slight differences due to the fact that higher statistics but simpler selection cuts compensate each other to a large extent. The upper limit of the fraction of gamma rays from the KASCADE measurement are presently the lowest upper limits, which are used to set constraints on theoretical predictions, in particular, on the distance of sources for the IceCube neutrino excess model [13]. By means of the KASCADE-Grande measurements, the best upper limit to the fraction of the gamma-ray to the cosmic-ray flux is obtained: $I_{\gamma} / I_{C R}<1.88 \cdot 10^{5}$ for 13.8 $\mathrm{PeV}$. The stringent limits above $100 \mathrm{PeV}$ might constrain a limit to the background rate of muon-poor showers in the search for the galactic disk enhancement of cosmic rays. A detailed analysis and discussion can be found in Ref.[14].

\section{KASCADE Cosmic ray Data Centre (KCDC)}

KCDC is a web portal (https://kcdc.ikp.kit.edu), where data of the KASCADE and KASCADE-Grande experiments are made available for the interested general public [15]. Since the first release in 2013, KCDC provides to the public users the measured and reconstructed parameters of air showers. In addition, KCDC provides the conceptual design, how the data can be treated and processed so that they are also usable outside the community of experts in the research field. Detailed educational examples make a use also possible for school students and early stage researchers. The aim of the project KCDC is the installation and establishment of a public data centre for high-energy 

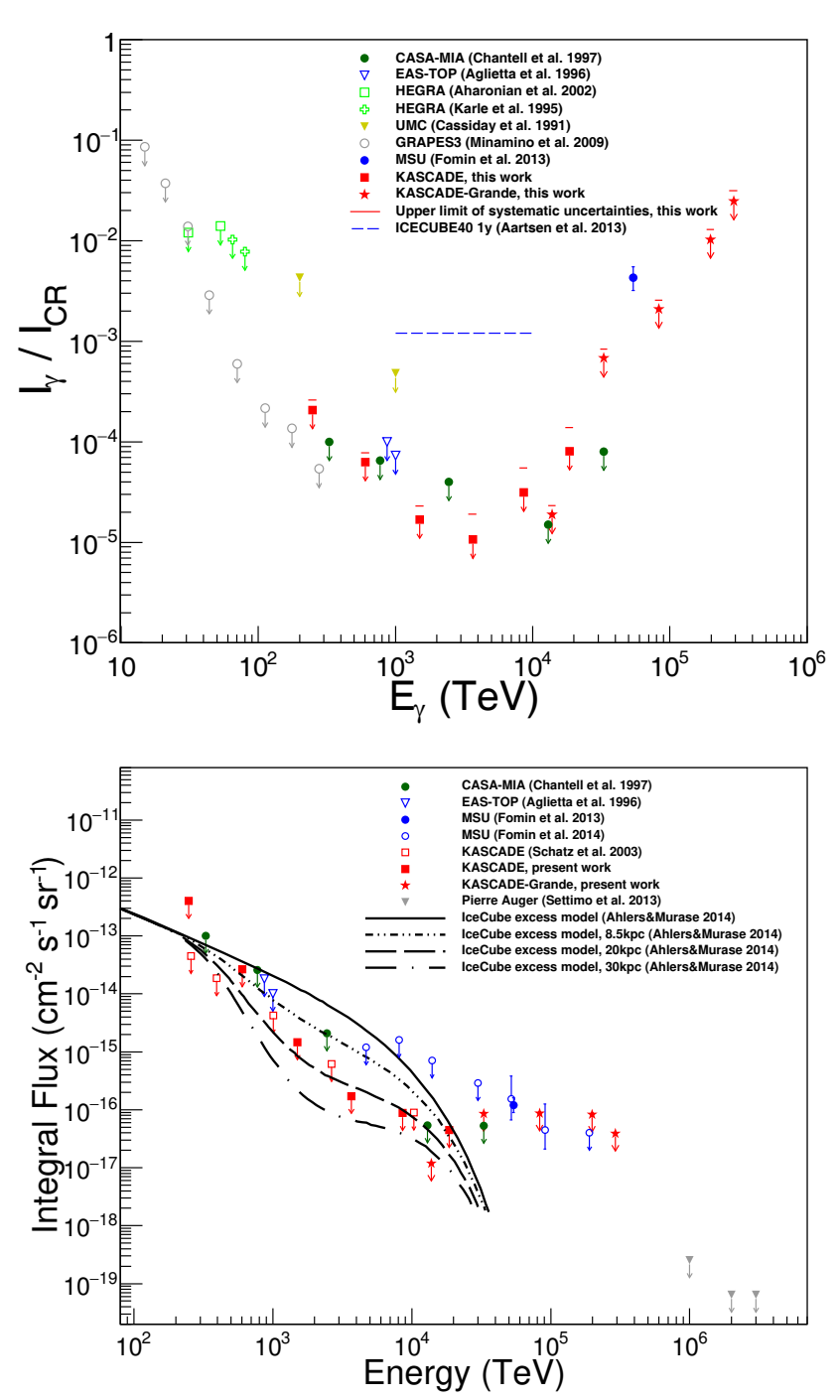

Figure 3. Top: Measurements of the fraction of gamma rays relative to cosmic rays in the energy from $10^{13} \mathrm{eV}$ to $10^{18} \mathrm{eV}$. The points with arrows represent upper limits from other experiments, except the MSU experimental value. The red squares and stars represent the results from KASCADE (90\% C.L.) and KASCADE-Grande (90\% C.L.), respectively, with systematic uncertainties. Bottom: Comparison of integral flux of gamma rays with previous results and with theoretical curves by an IceCube excess model [13].

astroparticle physics based on the data of the KASCADE experiment. Moreover, with KCDC we provide to the public the selected data via a custom-made web page.

In the new release of NABOO in 2007, data from the KASCADE-Grande detector component have been included to cover a larger part of the energy spectrum. $4.3 \cdot 10^{8}$ air shower events are available. For deeper investigations of the air-shower parameters, e.g. for composition analyses, full simulations of individual events are necessary. Thus we published also the full air-shower simulations with the inclusion of the detector responses. In addition, the data points of nearly 100 energy spectra from many different experiments were published as well.
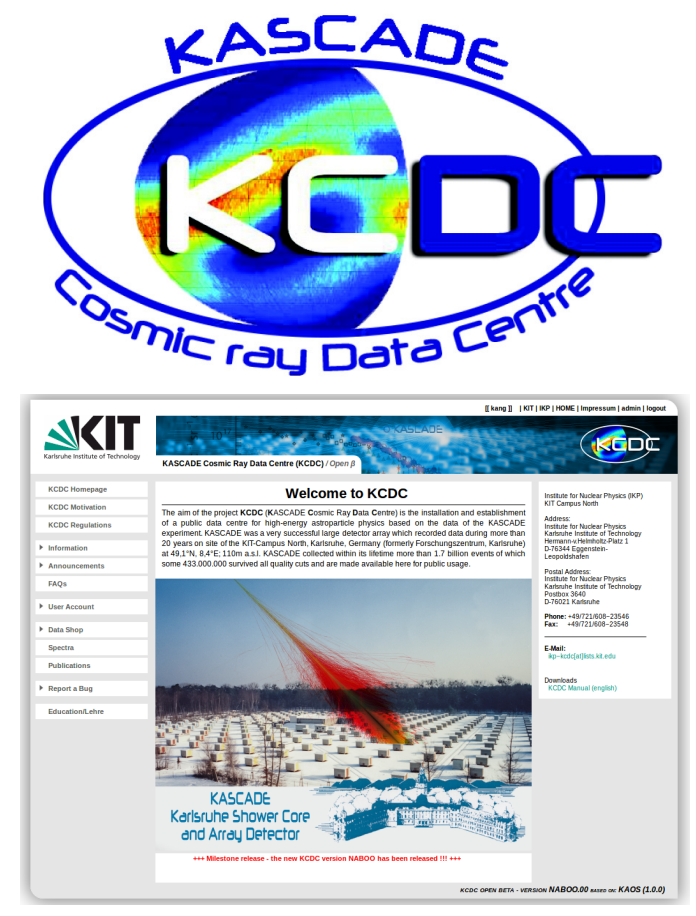

Figure 4. Logo of the KASCADE Cosmic-ray Data Centre (KCDC) in top and the index page of the website of KCDC, the KASCADE Cosmic-ray Data Centre, in bottom.

For the future, the publication of the accompanying software tools for open access will be achieved. Another plan for the future is to open KCDC for another type of shower data. Radio data from the LOPES experiment, which was co-located with KASCADE, will be included. Due to the different observation technique, the data structure from the LOPES antennas as well as calibration procedures are different from the ground-based KASCADE experiment, as well as the entire data analysis. Hence, an adoption of the data platform is required in direction of further generalization of KCDC.

\section{Conclusion}

All features of the energy spectra confirmed by the combined analysis of KASCADE and KASCADE-Grande: Observation of a 'heavy knee' at $9 \cdot 10^{16} \mathrm{eV}$ and Flattening of the light component around $10^{17} \mathrm{eV}$. It might be the first sign of an extra-galactic component. A validity of the most recent hadronic interaction model of SIBYLL $2.3 \mathrm{c}$ is tested, based on the shower size measurement by only KASCADE-Grande. The total energy flux is shifted by roughly $10 \%$, but all structures of energy spectra are similar. Using full data sets taken by KASCADE and KASCADE-Grande, the $90 \%$ C.L. upper limits to diffuse gamma-rays for energies of $200 \mathrm{TeV}$ to $300 \mathrm{PeV}$ are determined by selecting showers with low-muon contents. We obtained the best upper limit at 1.5 and $3.6 \mathrm{PeV}$, which constrains the IceCube excess model coming from $<20$ $\mathrm{kpc}$ in the galaxy. KCDC is a pioneering work in public access of astroparticle physics data and is already accepted 
by the astroparticle physics community. Since astroparticle physics experiments are globally distributed and the community requests for multi-messenger analyses, next steps of KCDC towards a global data and analysis centre for astroparticle physics are planned.

\section{References}

[1] T. Antoni et al., KASCADE Coll., Nucl. Instr. Meth. A513 (2003) 490

[2] W.D. Apel et al., KASCADE-Grande Coll., Nucl. Instr. and Meth. A620 (2010) 202

[3] T. Antoni et al., KASCADE Coll., Astropart. Phys. 24 (2005) 1-25

[4] W.D. Apel et al., KASCADE-Grande Coll., Astrop. Phys. 36 (2012) 183

[5] W.D. Apel et al., KASCADE-Grande Coll., Phys. Rev. Lett. 107 (2011) 171104
[6] W.D. Apel et al., KASCADE-Grande Coll., Phys. Rev. D 87 (2013) 081101

[7] S. Schoo, Doctoral Dissertation, KIT Karlsruhe (2016); DOI:10.5445/IR/1000055797

[8] J.C. Arteaga-Velázquez et al., KASCADE-Grande Coll., Astrop. Phys. 95 (2017) 25

[9] F. Riehn et al., PoS ICRC2017 (2017) 301

[10] D. Kang et al., KASCADE-Grande Coll., J. Phys.: Conf. Ser. 409 (2013) 012101

[11] O. Helene, Nucl. Instr. Meth. 212 (1983) 319

[12] G. Schatz et al. KASCADE Coll., Proc. 28th ICRC, Tsukuba, Japan (2003)

[13] M. Ahlers and K. Murase, Phys. Rev. D 90 (2014) 023010

[14] W.D. Apel et al. KASCADE-Grande Coll., Astropart. Phys. 848 (2017) 1

[15] A. Haungs, D. Kang et al., KASCADE-Grande Coll., Eur. Phys. J. C 78 (2018) 741 